\title{
METASTABILITY OF LOCALIZED NEUTRAL DONOR STATE IN GaAs*
}

\author{
C. Skierbiszewski ${ }^{\dagger}$, W. Jantsch, K. LüBKe \\ Johannes-Kepler-Universität, 4040 Linz, Austria
}

\section{Z. WILAMOWSKI}

Institute of Physics, Polish Academy of Sciences

Al. Lotników 32/46, 02-668 Warszawa, Poland

AND T. SUSKI

Unipress, Polish Academy of Sciences, Sokołowska 29, 01-142 Warszawa, Poland

Investigations of the photoconductivity of $\mathrm{GaAs}$ :Ge under hydrostatic pressure show, in addition to the well known persistent photoconductivity due to the DX state, another giant photoconductivity caused by a neutral localised " $\mathrm{A}_{1}$ " state of the donor. We find that the top of the barrier for the electron recapture to the $A_{1}$ state is pinned to the conduction band edge and the capture cross-section $\sigma(T \rightarrow \infty)$ is surprisingly small.

PACS numbers: 71.55. $-\mathrm{i}, 72.20$.Fr

It was established both experimentally [1, 2] and theoretically [3] that in addition to the DX donor state, a deep, localised one-electron state with the $A_{1}$ symmetry can exist in GaAs. The neutral charge of this state was also clearly evidenced [1]. There are however still some open questions concerning the microscopic model of the $A_{1}$ state, namely e.g., whether the localised state originates from central cell corrections only or it is accompanied by a strong lattice relaxation; whether and to what extent the $A_{1}$ state is hybridised with the effective mass states.

In this paper we report photoconductivity experiments performed in the temperature range of $10 \mathrm{~K}$ to $300 \mathrm{~K}$ and under hydrostatic pressure up to $20 \mathrm{kbar}$. During the Hall-effect and conductivity measurements the sample was illuminated

*This work is supported by FWF Lisa-Meitner Stipendium grant No. M074-PHY, FWF grant 10214 PHY and by grants Nos. 0550P49407, 2P30211705 of the State Committee for Scientific Research (Republic of Poland).

tPermanent address: Unipress, Polish Academy of Sciences, Sokołowska 29, 01-142 Warszawa, Poland. 
(with controlled intensity) by an infrared light emitting diode (IR LED) incorporated in the pressure cell filled with a liquid. We use metalorganic chemical vapour deposition (MOCVD) grown GaAs:Ge layers (thickness: $1.5 \mu \mathrm{m}$ ) grown on semi-insulating $\mathrm{Cr}$-doped GaAs substrate. The Ge-doping level varies from $10^{17}$ to $2 \times 10^{18} \mathrm{~cm}^{-3}$. These samples are semimetallic at low pressure. With increasing pressure the energy gap increases and the localised donor states ( $\mathrm{DX}$ and $\mathrm{A}_{1}$ ) move into the energy gap and become occupied [1].
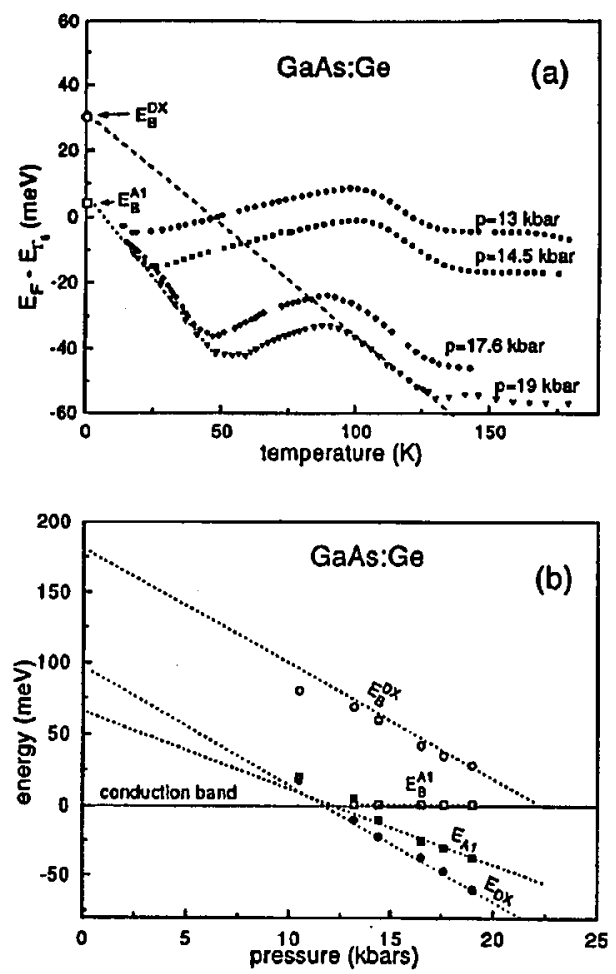

Fig. 1. (a) Temperature dependence of the Fermi level in GaAs:Ge, relative to the conduction band edge, for pressures: $13 \mathrm{kbar}(\bullet), 14.5 \mathrm{kbar}(\boldsymbol{\bullet}), 17.6 \mathrm{kbar}$ (full diamond), 19 kbar (full triangle). The dashed and dotted lines indicate the extrapolation used in order to evaluate the top of the barrier for the DX level, $E_{\mathrm{B}}^{\mathrm{DX}}(0)$ and for the $\mathrm{A}_{1}$ level, $E_{\mathrm{B}}^{\mathrm{A}_{1}}(\square)$ respectively. (b) Pressure dependencies of the DX (•), and the $A_{1}(\square)$ level and of the barriers for electron capture for the DX level $E_{\mathrm{B}}^{\mathrm{DX}}(0)$ and for the $\mathrm{A}_{1}$ level, $E_{\mathrm{B}}^{\mathrm{A}_{1}}(\square)$ respectively. Dotted lines are plotted as a guide for the eye.

In Fig. 1a, the temperature dependence of the quasi Fermi level $\left(E_{\mathrm{F}}^{*}\right)$, determined from the Hall concentration is shown relative to the conduction band (CB) edge under continuous illumination for different pressures for a sample with $n_{\mathrm{Ge}}=2 \times 10^{18} \mathrm{~cm}^{-3}$. The observed behaviour of $E_{\mathrm{F}}^{*}$ is typical of all investigated samples with a Ge concentration of $1 \times 10^{17}-2 \times 10^{18} \mathrm{~cm}^{-3}$ at pressures higher than 
$12 \mathrm{kbar}$. There are 4 characteristic temperature regimes which provide information about different details of the Ge related donors:

(i) At temperatures $T>140 \mathrm{~K}$ no metastability is observed. The system, including the DX states, is in thermal equilibrium there. There $E_{\mathrm{F}}^{*}$ is pinned to the DX state and thus the position of $E_{F}^{*}$ indicates the energy of the DX level, $E_{\mathrm{DX}}$. The pressure dependence of $E_{\mathrm{DX}}$ is shown by dots in Fig. $1 \mathrm{~b}$.

(ii) In the temperature range $75 \mathrm{~K}<T<140 \mathrm{~K}$, thermal emission from the DX state is much weaker than the optical one induced by the IR LED and $E_{\mathrm{F}}^{*}$ is established by the steady state equilibrium between optical emission of electrons from the DX state and the thermally activated capture of electrons to the DX state. It was shown by Jantsch et al. $[4,5]$ that under these conditions, a linear extrapolation of $E_{\mathrm{F}}^{*}$ to $T=0 \mathrm{~K}$ indicates the energy of the top of the barrier between $\mathrm{DX}$ and the neutral states (shallow and $\mathrm{A}_{1}$ state), $E_{\mathrm{B}}^{\mathrm{DX}}$. The positions of the $E_{\mathrm{B}}^{\mathrm{DX}}$ for different pressures, as obtained by a linear extrapolation towards zero temperature, is shown in Fig. $1 \mathrm{~b}$ by open circles.

(iii) For $50 \mathrm{~K}<T<80 \mathrm{~K}$, the thermal electron recapture to the DX states is already frozen and under illumination no DX states are occupied. Nevertheless, the temperature is still high enough to treat the subsystem of neutral donors and conduction electrons as remaining in equilibrium. Consequently, $E_{\mathrm{F}}^{*}$ is pinned to the $A_{1}$ level indicating its energetic position, $E_{\mathrm{A}_{1}}$. Proof for the neutral charge of this donor state was given in [1] and the name " $A_{1}$ " is inferred from the paper of Wasilewski et al. [6] and Dmochowski et al. [7]. The pressure variation of $E_{\mathrm{A}_{1}}$ is depicted by full squares in Fig. 1 b.

(iv) In the low temperature range an additional giant increase in photoconductivity is observed for pressures higher than $13 \mathrm{kbar}$, seen as an increase in $E_{\mathrm{F}}^{*}$ below $50 \mathrm{~K}$ in Fig. 1a. Switching the light off leads to a decrease in the electron concentration by orders of magnitude to values which correspond to an $E_{\mathrm{F}}^{*}$ located at the $\mathrm{A}_{1}$ state. For pressures higher than $14 \mathrm{kbar}$, low temperature measurements were possible only under illumination. The linear dependence of $E_{\mathrm{F}}^{*}$ vs. temperature results from the steady state equilibrium between optical emission from and recapture to the $A_{1}$ state. According to a similar approach as presented by Jantsch et al. for the DX case [4], extrapolation of $E_{\mathrm{F}}^{*}$ for $T=0 \mathrm{~K}$ indicates the top of the barrier for the $A_{1}$ state, $E_{\mathrm{B}}^{\mathrm{A}_{1}}$. The experimental points (see Fig. $1 \mathrm{~b}$ ) show that $E_{\mathrm{B}}^{\mathrm{A}_{1}}$ coincides with the $\mathrm{CB}$ edge and does not depend on pressure. The coincidence between $E_{\mathbf{B}}^{\mathbf{A}_{1}}$ and the CB edge suggests, however, that there is no substantial lattice barrier and the conduction electrons can be trapped practically without any activation energy. In such case the capture rate is described by the expression $\tau^{-1}=\sigma v_{\mathrm{th}} n_{\mathrm{D}+}$, where $\sigma$ - capture cross-section, $\tau$ - the time constant of the transient, $v_{\text {th }}$ - the thermal velocity of electrons and $n_{\mathrm{D}^{+}}$- the number of empty donor states (taking into account compensation). To evaluate whether electrons are captured from the $\mathrm{CB}$ or from electron traps, the temperature dependent kinetics was measured. The Arrhenius plot of the experimentally determined capture cross-section gives an activation energy of $5 \pm 2 \mathrm{meV}$ and $\sigma_{\exp }(T \rightarrow \infty) \approx 10^{-20} \mathrm{~cm}^{2}$. These two numbers show that the weakly temperature dependent capture - its activation energy is only of the or- 
der of $\mathrm{kT}$ in the accessible range - is impeded mostly by the apparently very small capture coefficient $\sigma_{\exp }(T \rightarrow \infty)$. Nevertheless, weak activation behaviour of $\sigma_{\text {exp }}$ indicates that the observed kinetics is affected by additional traps (located below the CB edge or mobility edge). When the concentration of the electrons on these traps $\left(n_{t}\right)$ is higher than the concentration of mobile electrons $\left(n_{c}\right)$, then the capture cross-section for the $A_{1}$ state, $\sigma$, is given by the following equation: $\sigma \approx \sigma_{\exp } \frac{n_{1}}{n_{\mathrm{c}}}$. This means that $\sigma$ is in the order of $10^{-17}-10^{-20} \mathrm{~cm}^{2}$ depending on $n_{\mathrm{t}}\left(n_{\mathrm{c}} \approx 10^{13}-10^{14} \mathrm{~cm}^{-3}\right.$, while $\left.n_{\mathrm{c}}<n_{\mathrm{t}}<10^{18} \mathrm{~cm}^{-3}\right)$. Whatever concentration of traps is assumed, this very rough approximation shows that the $A_{1}$ state has an extremely small capture cross-section.

In summary, we conclude that a new giant photoconductivity is caused by the $A_{1}$ state which exhibits an exceptionally small effective capture cross-section, indicative of some orthogonality of $\mathrm{CB}$ states and the $A_{1}$ symmetry donor states. Our results are in agreement with the finding of very weak anticrossing of the $A_{1}$ - and effective mass like states by Wasilewski [6].

\section{References}

[1] C. Skierbiszewski, T. Suski, P. Wis̉niewski, W. Jantsch, G. Ostermayer, Z. Wilamowski, P.G. Walker, N.J.. Mason, J. Singleton, Appl. Phys. Lett. 63, 3209 (1993).

[2] M. Baj, L. Dmowski, T. Słupiński, D. Wasik, Mater. Sci. Forum 143-147, 1019 (1994).

[3] J. Dabrowski, M. Scheffler, R. Strehlow, in: Proc. ICPS-20, Eds. E.M. Anastassakis, J.D. Joannopoulos, World Scientific, Singapore 1990, p. 489.

[4] W. Jantsch, Z. Wilamowski, G. Ostermayer, Semicond. Sci. Technol. 6, B47 (1991).

[5] Z. Wilamowski, W. Jantsch, G. Ostermayer, J. Kossut, Mater. Sci. Forum 83-87, 805 (1992).

[6] Z. Wasilewski, R.A. Stradling, Semicond. Sci. Technol. 1, 264 (1986).

[7] J. Dmochowski, Z. Wasilewski, R.A. Stradling, Mater. Sci. Forum 65-66, 449 (1990). 